\title{
Three dimensional characterization of interfaces using aberration-corrected STEM
}

K. van Benthem ${ }^{1}$, A.Y. Borisevich ${ }^{1}$, M.F. Chisholm ${ }^{1}$, A.R. Lupini ${ }^{1}$, S.T. Pantelides $^{2,1}$, S. Rashkeev ${ }^{1,2}$, M. Varela ${ }^{1}$, S.J. Pennycook ${ }^{1}$

1 Oak Ridge National Laboratory, Condensed Matter Science Division, Oak Ridge, TN 37831-6031, USA

2 Department of Physics and Astronomy, Vanderbilt University, Nashville, TN 37235, USA

The distribution of single dopant atoms can dramatically alter the properties of functional nano-materials. The detectability and lateral localization of such single atoms has been greatly improved by the recent development of aberration correction in scanning transmission electron microscopy (STEM), which enables the formation of electron probes with diameters that can be as small as $0.06-0.08$ $\mathrm{nm}[1,2]$. By focusing the electron probe into different planes of the specimen, a slice-by-slice image of three-dimensional structures is available, which is realized by recording through-focal series (see Figure 1). Recorded image stacks can then be used for 3-D reconstructions.

Figure 2a shows an annular dark-field image of a $\mathrm{HfO}_{2} / \mathrm{SiO}_{2} / \mathrm{Si}$ interface recorded with the aberration-corrected VG Microscopes HB603U STEM [2]. Isolated $\mathrm{Hf}$ atoms are observable in the amorphous $\mathrm{SiO}_{2}$ layer and are marked in the Figure. Figure $2 \mathrm{~b}$ shows a $3 \mathrm{D}$ reconstruction of the $\mathrm{HfO}_{2} / \mathrm{SiO}_{2} / \mathrm{Si}$ interface. $\mathrm{No} \mathrm{Hf}$ atom embedded in the $\mathrm{SiO}_{2}$ interlayer was found to be closer than 0.25$0.30 \mathrm{~nm}$ to the $\mathrm{Si} / \mathrm{SiO}_{2}$ interface. Total energy calculations using density functional theory [3] support this observation and allow a detailed analysis of the local atomic arrangements and the local band structure.

Aberration-corrected electron energy-loss spectroscopy was applied to the interface to investigate the local unoccupied densities of states and the complex dielectric function $\varepsilon=\varepsilon_{1}+i \varepsilon_{2}$ locally across the gate dielectric interface. Hence, physical properties, such as local dielectric constants, local indexes of refraction and band gap energies can be determined. Examples will be shown in the presentation.

[1] P.E. Batson, N. Dellby, O.L. Krivanek, Nature 418, 617-620 (2002).

[2] P.D. Nellist et al., Science 305, 1741 (2004).

[3] S. N. Rashkeev, D. M. Fleetwood, R. D. Schrimpf, and S. T. Pantelides, Phys. Rev. Lett. 87, 1655061 (2001).

[4] Financial support from the Alexander-von-Humboldt Foundation is gratefully acknowledged. 


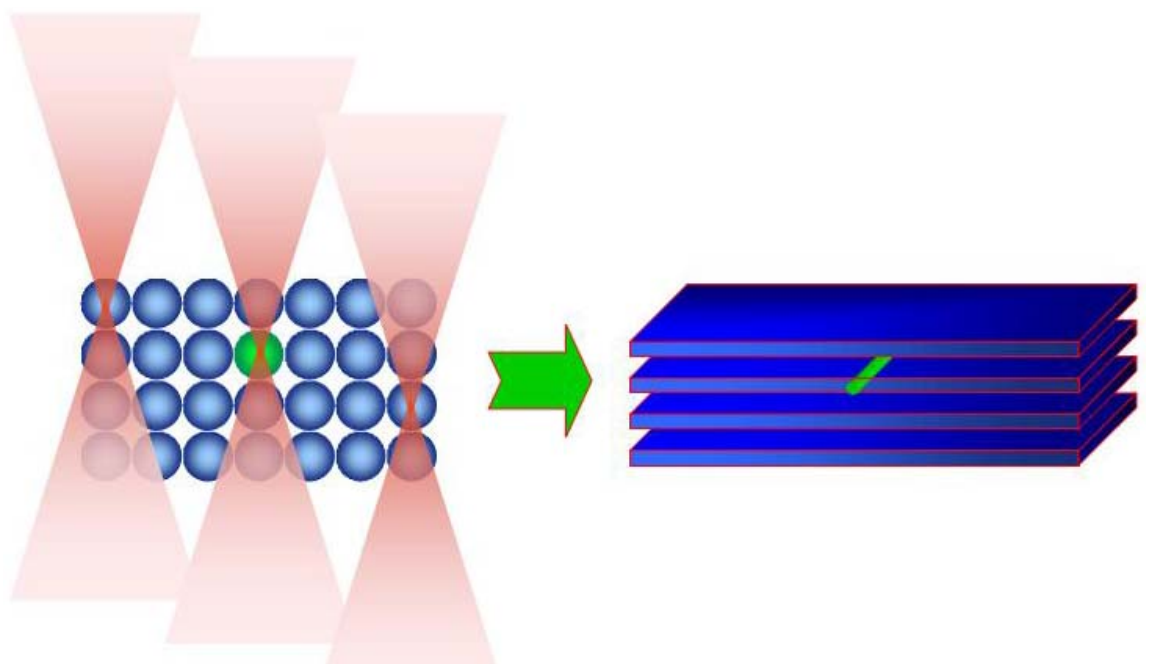

Figure 1: Focusing the electron probe into different planes of the specimen produces a slice-by-slice image of a threedimensional structure. The acquired data sets can then be used for 3D reconstructions.
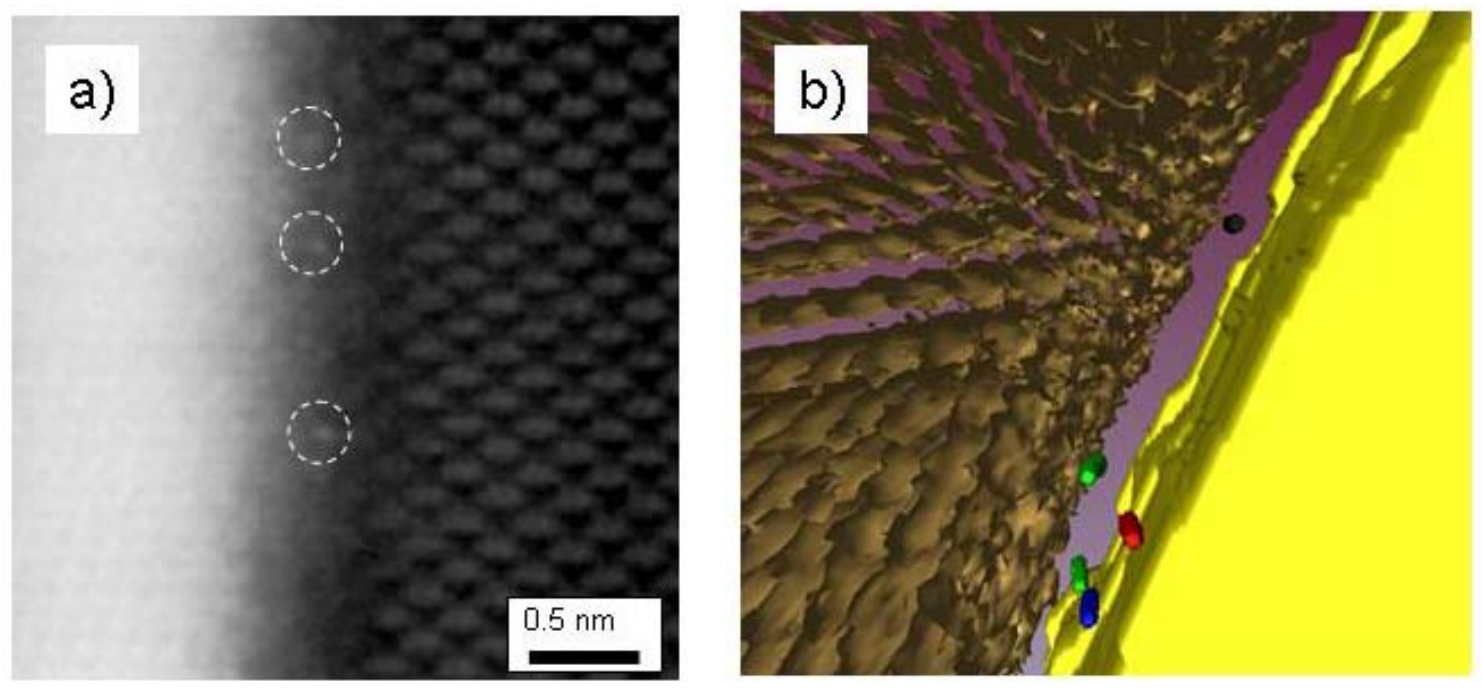

Figure 2: a): High-angle annular dark-field image of a $\mathrm{HfO}_{2} / \mathrm{SiO}_{2} / \mathrm{Si}$ gate dielectric interface. Numerous isolated $\mathrm{Hf}$ atoms are found in the $\mathrm{SiO}_{2}$ interlayer at different depths. Two of the isolated atoms which are in focus in the shown frame are marked in the image.

b): Reconstructed three-dimensional structure model of the $\mathrm{HfO}_{2} / \mathrm{SiO}_{2} / \mathrm{Si}$ interface. Some of the isolated $\mathrm{Hf}$ atoms are represented by green, blue, red, and black colors. The HfO2 film is shown in bold yellow, the Si substrate in gold. 